\title{
Elektrochemotherapie zur Behandlung von Hauttumoren und Hautmetastasen
}

\author{
Electrochemotherapy as a Treatment for Skin Tumors and Metastases
}

Autoren

Institut
J. Kirschke, S. Knispel, J. Hyun, C. Tigges, A. Kreuter

Klinik für Dermatologie, Venerologie und Allergologie, HELIOS St. Elisabeth Klinik, Oberhausen
Bibliografie

DOI http://dx.doi.org/

10.1055/s-0034-1365519

Online-Publikation: 10.4.2014

Akt Dermatol 2014; 40: 177-180

(c) Georg Thieme Verlag KG

Stuttgart · New York

ISSN 0340-2541

Korrespondenzadresse

Prof. Dr. med.

Alexander Kreuter

Chefarzt der Klinik für

Dermatologie, Venerologie

und Allergologie

HELIOS St. Elisabeth Klinik

Josefstr. 3

46045 Oberhausen

alexander.kreuter@helios-

kliniken.de

\section{Zusammenfassung \\ $\nabla$}

Nach Ausschöpfung von klassischen Behandlungsverfahren wie Chemo- und Strahlentherapie gibt es für Patienten mit ausgedehnten, inoperablen Hauttumoren oder Hautmetastasen nur wenige Möglichkeiten einer lokalen Tumorkontrolle. Die Elektrochemotherapie bietet in diesen Fällen eine hocheffektive, nebenwirkungsarme Behandlungsoption, die wiederholt auch in vorbehandelten

\section{Einleitung \\ $\nabla$}

Die Elektrochemotherapie wurde gegen Ende der 1980er-Jahre erstmalig in Frankreich als neue Behandlungsoption beschrieben [1]. Es konnte gezeigt werden, dass durch die Anlage eines elektrischen Spannungsfeldes eine kurzfristige Öffnung der Zellmembran (sog. Elektroporation) erfolgt, wodurch Zellen um ein Vielfaches durchlässiger für Moleküle werden, die ansonsten nicht oder nur in sehr geringem Maße in diese penetrieren können [2-4]. Die Elektrochemotherapie wurde zunächst im Rahmen von präklinischen Studien mit unterschiedlichen Chemotherapeutika an Hautmetastasen erprobt. Hierbei konnte eine bis zu 80-fache Steigerung der anti-tumoralen Wirkung für Cisplatin und eine bis zu 8000-fache Steigerung für Bleomycin nachgewiesen werden $[3,5,6]$. Im Anschluss daran wurde die Elektrochemotherapie mit Bleomycin dann erstmals im Jahr 1991 im Rahmen von klinischen Studien an Patienten mit Plattenepithelkarzinomen der Kopf-Hals-Region durchgeführt. Hierbei zeigten sich Ansprechraten von bis zu 72\% und eine komplette Rückbildung bei mehr als der Hälfte der Tumore [5]. Weitere Folgestudien untersuchten den Einsatz der Elektrochemotherapie bei einer Vielzahl unterschiedlicher Hauttumore und kutaner Metastasen wie Plattenepithelkarzinom der Haut, malignem Melanom ( $\bullet$ Abb. 1 und - Abb.2) oder kutane Metastasen bei Mamma-
Arealen und ohne wesentliche Einschränkungen im Hinblick auf Anzahl und Lage der Metastasen eingesetzt werden kann. Durch die Reduktion der Tumormasse sowie Minderung etwaiger Blutungen und Exsudation kann eine deutliche Verbesserung der Lebensqualität für die betroffenen Patienten erzielt werden. Der Erfolg der Elektrochemotherapie gibt aktuell Anlass zur Überprüfung der Indikationserweiterung auf viszerale Tumore, Knochenmetastasen und sogar Hirntumore.

karzinom [2]. Um den Einsatz der Elektrochemotherapie mit Bleomycin oder Cisplatin bei kutanen und subkutanen Malignom-Metastasen zu standardisieren, wurde 2003 eine internationale Studie (European Standard Operating Procedures of the Electrochemotherapy, ESOPE) durchgeführt und ein einheitliches Behandlungsprotokoll festgelegt [7]. Im Jahre 2008 wurde die Elektrochemotherapie auch in Deutschland eingeführt und wird seither zunehmend als wirkungsvolle und nebenwirkungsarme Behandlungsoption bei kutanen und subkutanen Hautmetastasen verschiedenster Tumorentitäten eingesetzt.

\section{Wirkprinzip der Elektrochemotherapie $\nabla$}

Mit Hilfe speziell für die Elektrochemotherapie entwickelter Elektroden wird ein elektrisches Feld im Tumorgewebe erzeugt. Hierbei kommt es zu einer vorübergehenden Entwicklung von sogenannten Elektroporen, durch die die Zellmembran um ein Vielfaches durchgängiger für sonst nur schwer oder nicht permeable Moleküle gemacht wird ( $\bullet$ Abb.3). Zu solchen Molekülen zählen bestimmte Chemotherapeutika wie insbesondere Bleomycin und Cisplatin, die durch diese Poren in erhöhtem Maße in die Tumorzellen penetrieren können [8,9]. Über diesen Mechanismus kann im Vergleich zu einer klassischen Chemotherapie mit einer deutlich geringeren Dosie- 


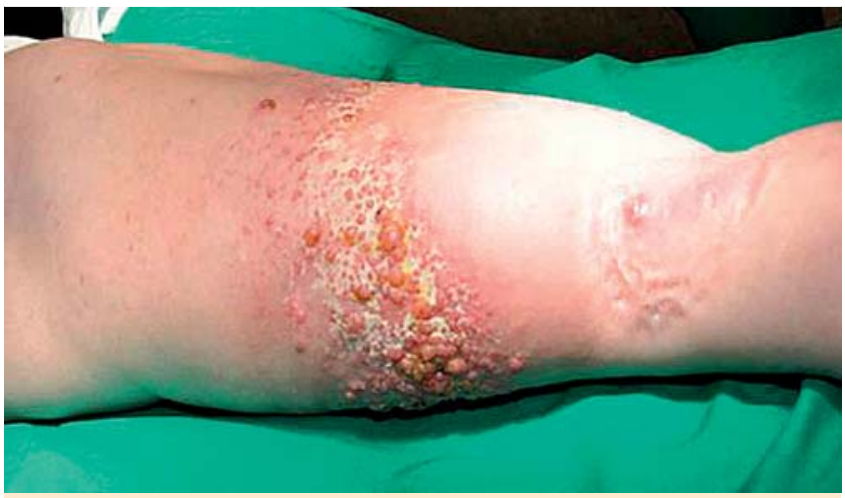

Abb. 1 Ausgedehnte, flächig imponierende kutane Metastasierung des rechten Unterschenkels bei malignem Melanom.

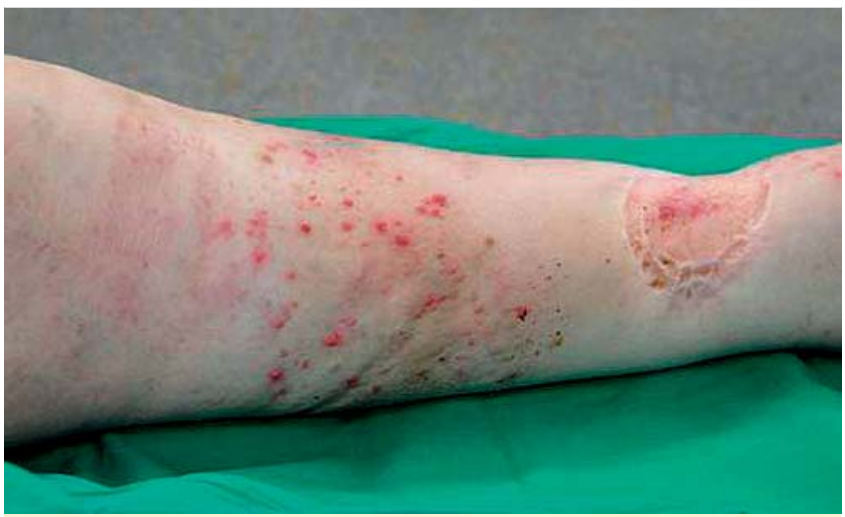

Abb.2 Deutliche Reduktion der Tumorknoten und Reduktion des tumorbedingten Ödems nach Elektrochemotherapie.



Abb. 3 Schematische Darstellung der Elektroporation. Phase 1: Injektion des Zytostatikums (intravenös oder intraläsional). Phase 2: Elektroporation: Das Chemotherapeutikum gelangt in die Tumorzelle (bis zu 10000-fach höher konzentriert als bei konventioneller intravenöser Gabe). Phase 3: Reversible Poration: Die Zellmembran schließt sich, das Chemotherapeutikum beginnt zu wirken (Quelle: IGEA GmbH, Clinical Biophysics, Frankfurt am Main). rung eine vielfach höhere Wirkung im Tumorgewebe erzielt werden. Für andere Zytostatika wie Doxorubicin, Adriamycin, Melphalan, Methotrexat, Paclitaxel, Vinblasin, Vincristin, 5-Fluorouracil oder Mitomycin C konnte nach Elektroporation in vitro jedoch keine erhöhte Penetration nachgewiesen werden [3]. Als weiterer Effekt der Elektrochemotherapie kommt es zu einer kurzfristigen Reduktion des Blutflusses im Behandlungsareal, die nach der Applikation des elektrischen Impulses bis zu 24 Stunden fortbestehen kann. Somit verbleibt das verwendete Zytostatikum länger am Applikationsort und kann entsprechend länger seine Wirkung entfalten. Weiterhin kommt es im Zuge der verminderten Durchblutung zu einer tumoralen Hypoxie, was wiederum zum Untergang von Tumorzellen führt $[10,11]$. Die Zerstörung der Tumorzellen mit nachfolgender Freisetzung von Tumorantigenen führt zudem zu einer breiten Immunstimulation, die das Gesamtansprechen der Elektrochemotherapie weiter erhöht [2].

\section{Durchführung der Elektrochemotherapie \\ $\nabla$}

Die Elektrochemotherapie wird entsprechend dem oben erwähnen Behandlungsprotokoll der ESOPE durchgeführt [7]. Der Eingriff findet in aller Regel in kurzer Intubationsnarkose statt. Bei weniger schwer ausgeprägten Befunden oder Kontraindikationen gegen eine Vollnarkose ist auch der Einsatz in Tumeszenzanästhesie möglich. Primär werden als Chemotherapeutika Bleomycin und Cisplatin verwendet. Bei Einsatz von Bleomycin ist neben einer intravenösen Gabe auch die intraläsionale Applikation möglich. Die Dosierung der Medikation hängt bei der intraläsionalen Applikation vom Tumorvolumen, bei der systemischen Applikation von der Körperoberfläche des Patienten ab. Nach Gabe der Chemotherapie wird in einem Zeitraum von einer Minute nach intraläsionaler bzw. etwa acht Minuten nach intravenöser Gabe die Elektroporation begonnen. Die elektrischen Impulse können je nach Größe und Form des Tumors mittels verschiedener Arten von Elektroden (hexagonal oder linear) verabreicht werden. Diese dringen etwa ein bis drei Zentimeter tief in das Tumorgewebe ein. Die komplette Behandlung sollte innerhalb eines therapeutischen Zeitfensters von etwa 25 Minuten abgeschlossen sein, da es nachfolgend zu einem kontinuierlichen Wirkungsverlust kommt. Bei sehr ausgedehnten Tumorknoten, Rezidiven oder unvollständiger Rückbildung der Tumore nach Durchführung der Elektrochemotherapie sind weitere Folgesitzungen möglich.

\section{Primäre Indikationen der Elektrochemotherapie} $\nabla$

Die Elektrochemotherapie kann prinzipiell bei allen kutanen oder subkutanen Metastasen in Erwägung gezogen werden, die aufgrund der Ausdehnung, Lokalisation oder Vortherapie operativ nicht oder nur schwer behandelt werden können und/oder bei denen systemische Chemotherapien bzw. Bestrahlungstherapien unwirksam waren oder für den Patienten zu belastend sind. Das primäre Einsatzgebiet der Elektrochemotherapie liegt aktuell vor allem in der Therapie von kutanen und subkutanen Melanom- und Mammakarzinom-Metastasen [12]. In zahlreichen Studien und Berichten konnte aber bereits die Wirksamkeit für diverse andere Tumorentitäten aufgezeigt werden. Dazu zählen unter anderem Plattenepithelkarzinome der Haut, Kopf-HalsKarzinome (z.B. Oro- und Hypopharynxkarzinome), urogenitale 


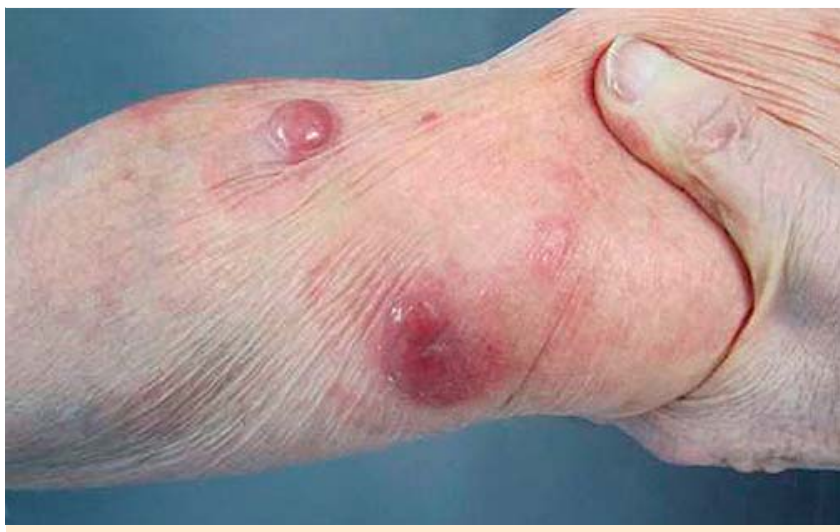

Abb.4 Multipel vorbehandeltes kutanes T-Zell-Lymphom vom Typ der Mycosis fungoides im Tumorstadium im Bereich des linken Unterarmes.

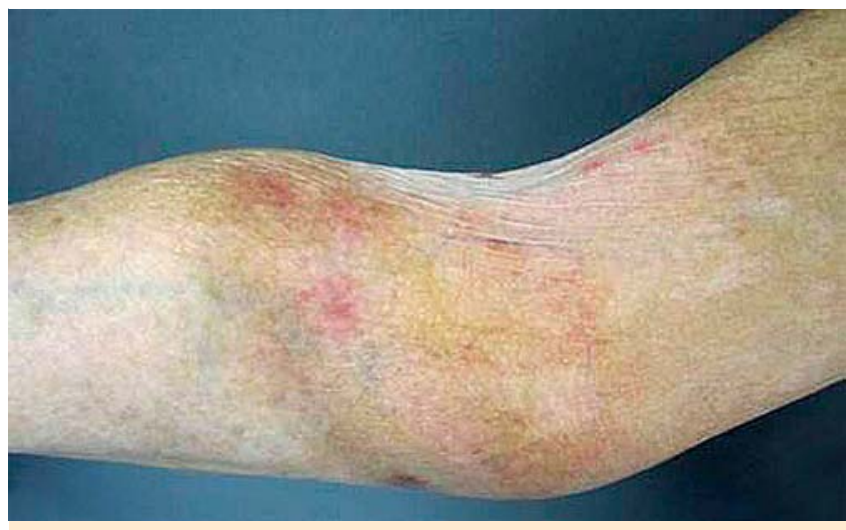

Abb.5 Komplette Abheilung aller Tumorknoten nach Elektrochemotherapie.

Karzinome, kutane Lymphome ( Abb.4 und $\bullet$ Abb.5), Merkelzellkarzinome, Angiosarkome, Kaposi-Sarkome, Speicheldrüsenkarzinome und Sarkome [13].

\section{Erweiterte Indikationen der Elektrochemotherapie $\nabla$}

In präklinischen Studien führte die lokale Anwendung von Interleukin-2 zu gesteigerten Remissionsraten, sodass in der Zukunft die Kombination der Elektrochemotherapie mit entsprechenden Immuntherapien denkbar ist [14-18]. Neben der Kombination mit Immuntherapien sind auch Indikationserweiterungen auf viszerale Tumore und Metastasen wie zum Beispiel Leberfiliae, Hirnfiliae und Knochenmetastasen Gegenstand aktueller Forschung $[19,20]$. Ebenso sind aktuell verschiedene alternative Applikationssonden in Erprobung. Dazu gehört zum einen die sogenannte „long needle“, welche bei Leber- und Knochenmetastasen sowie Weichteilsarkomen erprobt wird [21]. Sinnvoll könnte zudem die Anwendung bei inoperablen hepatozellulären Karzinomen und Cholangiokarzinomen sein [3]. In Irland wurde eine endoluminale Elektrode bei kolorektalen, gastralen und ösophagealen Tumoren erfolgreich angewendet [21]. In Dänemark findet die sogenannte expandierbare, schirmartige (sog. „umbrella“ type) Sonde bei Hirntumoren Anwendung. Diese kann in das Tumorareal eingebracht werden, ohne dass eine Kraniotomie durchgeführt werden muss [22]. Aktuell wird vor allem die Sicherheit von viszeral oder intrakraniell applizierten Sonden ge- prüft. Auch die Wechselwirkungen der elektrischen Ströme mit dem Reizleitungssystem des Herzmuskels sind bei anatomischer Nähe (z.B. Brustwandtumore) zu beachten. Eine mögliche Option ist die Synchronisation der elektrischen Impulse mit der elektrischen Aktivität des Herzmuskels, sodass Erregungen während der vulnerablen Phase des Erregungszykluses ausgeschlossen werden $[23,24]$.

\section{Mögliche Nebenwirkungen unter Elektrochemo- therapie}

Die häufigsten Nebenwirkungen unter einer Elektrochemotherapie sind muskelkaterartige Beschwerden, die durch die unwillkürlichen Muskelkontraktionen unter der Impulsabgabe hervorgerufen werden [25]. Derartige Muskelschmerzen sind jedoch unter einer entsprechenden Analgesie sehr gut beherrschbar. Des Weiteren kommt es, abgesehen von den sichtbaren Elektroden-Einstichstellen, gehäuft zu Lokalreaktionen in Form von Rötung, Juckreiz, Schwellung, Blasenbildung und Brennen sowie möglicherweise zu Pigmentveränderungen und überschießender Narbenbildung. Auch die Entstehung oberflächlicher bis tiefer Nekrosen im Bereich der behandelten Tumorherde kann auftreten. Derartige Nebenwirkungen weisen jedoch auf ein gutes Therapieansprechen hin und lassen sich unter konsequenter Lokaltherapie zur Abheilung bringen. Da die Elektrochemotherapie ihre Wirkung vor allem auf schnell proliferierende Krebszellen ausübt, sind Nebenwirkungen im Bereich des umgebenden gesunden Gewebes meist nur gering ausgeprägt. Weitere mögliche Nebenwirkungen treten unabhängig von der Elektroporation auf und sind auf das herkömmliche Nebenwirkungsprofil der Chemotherapeutika zurückzuführen. Dazu zählen in seltenen Fällen Fieber, Nausea und Emesis sowie Arthralgien und Myalgien. Ferner können Mukositis, Alopezie und Nagelwachstumsstörungen auftreten. Über einer Gesamtdosis von $300 \mathrm{mg}$ Bleomycin kann es zudem zur Lungenfibrose kommen. Eine nicht seltene, Bleomycin-typische kutane Nebenwirkung ist die Flagellanten-Dermatitis. Diese Chemotherapie-bedingten Nebenwirkungen treten aufgrund der nur geringen Dosierung bei der Elektrochemotherapie in einem deutlich geringeren Anteil als bei einer herkömmlichen Chemotherapie auf.

\section{Anwendungsbeschränkung/Kontraindikationen für die Elektrochemotherapie \\ $\nabla$}

Die im Rahmen der Elektrochemotherapie abgegebenen elektrischen Impulse können bei Patienten mit implantierten Schrittmachern zu Fehlfunktionen und Gewebeschädigungen führen. Weiterhin ist das Einbringen der Elektroden im Bereich implantierter Portsysteme gefährlich, da dies eine Verletzungen von Gefäßen oder des Katheters bedingen kann.

\section{Ausblick \\ $\nabla$}

Die derzeitige Indikation zum Einsatz der Elektrochemotherapie umfasst Patienten in palliativer Gesamtsituation oder inoperable Tumore, die durch die Anwendung anderer Verfahren nicht lokal kontrolliert werden konnten. Die aktuelle klinische Forschung erprobt die Erweiterung der Elektrochemotherapie auf viszerale und tiefer liegende inoperable Tumore [26,27]. Ob und bei 
welchen Tumoren die Elektrochemotherapie in Zukunft auch als Verfahren bei kurativer Zielsetzung Anwendung findet und mit anderen Verfahren wie der Radiofrequenzablation oder Chirurgie konkurrieren kann, bleibt unklar. Hierzu fehlen bis dato noch kontrollierte klinische Studien.

\section{Fazit}

$\nabla$

Die Elektrochemotherapie bietet in Fällen ausgedehnter Hautmetastasen verschiedener solider Tumoren sowie primär kutaner Neoplasien eine hocheffektive, nebenwirkungsarme Behandlungsoption, welche wiederholt auch in vorbehandelten Arealen und ohne wesentliche Einschränkungen im Hinblick auf Anzahl und Lage der Metastasen eingesetzt werden kann. Durch die Reduktion der Tumormasse sowie Minderung von Blutungen und Exsudation kann eine deutliche Besserung der Lebensqualität für die betroffenen Patienten erzielt werden. Der Erfolg der Elektrochemotherapie gibt aktuell Anlass für die Überprüfung der Indikationserweiterung auf viszerale Tumore, Knochenmetastasen und sogar Hirntumore.

\section{Interessenkonflikt}

$\nabla$

Die Autoren geben an, dass kein Interessenkonflikt besteht.

Abstract

\section{Electrochemotherapy as a Treatment for Skin Tumors and Metastases \\ $\nabla$}

Following classic treatment options for cancer patients such as chemotherapy or radiation, only few therapies remain for patients with widespread, inoperable skin tumours or metastases. Electrochemotherapy is a highly effective treatment approach in such cases with few side effects. Electrochemotherapy can be repeatedly performed even in pre-treated areas without substantial limitations concerning number and location of skin metastases. Reduction of tumor mass and decrease of bleeding and exudation might significantly improve patient's quality of live. The overall success of electrochemotherapy encourages expanding its indication to visceral tumours or bone and brain metastases.

\section{Literatur}

1 Mir LM, Orlowski S, Belehradek JJr et al. Electrochemotherapy potentiation of antitumour effect of bleomycin by local electric pulses. Eur J Cancer 1991; 27: 68-72

2 Reinhold U. Electrochemotherapy of skin tumors. Hautarzt 2011; 62: $549-559$

3 Miklavčič D, Mali B, Kos B et al. Electrochemotherapy: from the drawing board into medical practice. Biomed Eng Online 2014; 13: 29

4 Okino M, Mohri H. Effects of a high-voltage electrical impulse and an anticancer drug on in vivo growing tumors. Jpn J Cancer Res 1987; 78: $1319-1321$

5 Sersa G, Stabuc B, Cemazar M et al. Electrochemotherapy with cisplatin: potentiation of local cisplatin antitumour effectiveness by application of electric pulses in cancer patients. Eur J Cancer 1998; 34: 1213 1218
6 Sersa G. Cemazar M, Miklavcic D. Antitumor effectiveness of electrochemotherapy with cis-diamminedichloroplatinum (II) in mice. Cancer Res 1995; 55: 3450 - 3455

7 Mir LM, Gehl J, Sersa G et al. Standard operating procedures of the electrochemotherapy: instructions for the use of bleomycin or cisplatin administered either systematically or locally and electric pulses delivered by the Clinicoporator by means of invasive or non-invasive electrodes. Eur J Cancer 2006; 4: 14-25

8 Pakhomov AG, Shevin R, White JA et al. Membrane permeabilization and cell damage by ultrashort electric field shocks. Arch Biochem Biophys 2007; 465: 109-118

9 Pucihar G, Kotnik T, Miklavcic D et al. Kinetics of transmembrane transport of small molecules into electropermeabilized cells. Biophys J 2008; 95: 2837-2848

10 Markelc B, Sersa G, Gemazar M. Differential mechanisms associated with vascular disrupting action of electrochemotherapy: intravital microscopy on the level of single normal and tumor blood vessels. PloS One 2013; 8: e59557

11 Sersa G, Jarm T, Coer A et al. Vascular disrupting action of electroporation and electrochemotherapy with bleomycin in murine sarcoma. $\mathrm{Br} \mathrm{J}$ Cancer 2008; 98: 388 - 398

12 Matthiessen LW, Johannesen HH, Hendel HW et al. Electrochemotherapy for large cutaneous recurrence of breast cancer: A phase II clinical trial. Acta Oncol 2012; 51: 713-721

13 Sersa $G$. The state-of-the-art of electrochemotherapy before ESOPE study; advantages and clinical uses. Eur J Cancer Suppl 2006; 4: 52 - 59

14 Hyacinthe $M$, Jaroszeski MJ, Dang $V$ et al. Electrically enhanced drug delivery for the treatment of soft tissue sarcoma. Cancer 1999; 85: 409-417

15 Sersa G, Cemazar M, Menart Vet al. Anti-tumor effectiveness of electrochemotherapy with bleomycin is increased by TNF-alpha on SA-1 tumors in mice. Cancer Lett 1997; 116: 85-92

16 Heller L, Pottinger C, Jaroszeski MJ et al. In vivo electroporation of plasmids encoding GM-CSF or interleukin-2 into existing B16 melanomas combined with electrochemotherapy induces long-term antitumour immunity. Melanoma Res 2000; 10: 577-583

17 Daud A, DeConti R, Andrews $S$ et al. Phase I trial of interleukin-12 plasmid electroporation in patients with metastatic melanoma. J Clin Oncol 2008; 26: 5896 - 5903

18 Sedlar A, Dolinsek T, Markelc B et al. Potentiation of electrochemotherapy by intramuscular IL-12 gene electrotransfer in murine sarcoma and carcinoma with different immunogenicity. Radiol Oncol 2012; 46: $302-311$

19 Edhemovic I, Gadzijev EM, Brecelj E et al. Electrochemotherapy: a new technological approach in treatment of metastases in the liver. Technol Cancer Res Treat 2011; 10: 475-485

20 Miklavcic D, Sersa G, Brecelj E et al. Electrochemotherapy: technological advancements for efficient electroporation-based treatment of internal tumors. Med Biol Eng Comput 2012; 50: 1213-1225

21 Soden DM, Larkin JO, Collins CG et al. Successful application of targeted electrochemotherapy using novel flexible electrodes and low dose bleomycin to solid tumours. Cancer Lett 2006; 232: 300-310

22 Linnert M, Iversen HK, Gehl J. Multiple brain metastases - current management and perspectives for treatment with electrochemotherapy. Radiol Oncol 2012; 46: 271-278

23 Deodhar A, Dickfeld T, Single GW et al. Irreversible electroporation near the heart: ventricular arrhythmias can be prevented with ECG synchronization. AJR Am J Roentgenol 2011; 196: 330-335

24 Mali B, Jarm T, Corovic $S$ et al. The effect of electroporation pulses on functioning of the heart. Med Biol Eng Comput 2008; 46: 745-757

25 Zupanic A, Ribaric S, Miklavic D. Increasing the repetition frequency of electric pulse delivery reduces unpleasant sensations that occur in electrochemotherapy. Neoplasma 2007; 54: 246-250

26 Bianchi G, Campanacci L, Donati D. Electrochemotherapy Treatment of Locally Advanced and Metastatic Soft Tissue Sarcomas: Results of a Non-Comparative Phase II Study. World J Surg 2014; 38: 813-822

27 Mahmood F, Gehl J. Optimizing clinical performance and geometrical robustness of a new electrode device for intracranial tumor electroporation. Bioelectrochemistry 2011; 81: 10-16 